\title{
Benidipine reduces ischemia reperfusion-induced systemic oxidative stress through suppression of aldosterone production in mice
}

\author{
Keisuke Ohtani, Soichiro Usui, Shuichi Kaneko, Shin-ichiro Takashima, Katsunori Kitano, Kanako Yamamoto,
} Masaki Okajima, Hiroshi Furusho and Masayuki Takamura

\begin{abstract}
Aldosterone is implicated in the pathogenesis of several cardiovascular diseases, including ischemia reperfusion (I/R) and myocardial infarction, and also causes oxidative stress and inflammation in cardiovascular systems. Benidipine, a long-acting T- and L-type calcium channel blocker, reduces infarct size following myocardial $I / R$ in rabbits. Benidipine also inhibits the production of aldosterone in vitro. However, the precise mechanism of this phenomenon in vivo remains unknown. We therefore evaluated whether benedipine has a beneficial role through the regulation of oxidative stress in myocardial I/R. C57BL/6J mice were subjected to $30 \mathrm{~min}$ of left ascending coronary I/R. Benidipine was administered orally at $3 \mathrm{mg} \mathrm{kg}^{-1}$ daily for 3 weeks without any changes in hemodynamic variables. Benidipine significantly reduced infarction size $(13.4 \pm 2.5 \%)$ compared with controls $(25.5 \pm 3.6 \%)$. Urinary 8-hydroxy-2' deoxyguanosine (8-OHdG), a marker of oxidative DNA damage, increased significantly after I/R. I/R induced increases in 8-OHdG were significantly lower with benidipine. Local myocardial 8-OHdG was also elevated in $\mathrm{I} / \mathrm{R}$, but this augmentation was significantly suppressed with benidipine. The plasma aldosterone concentration (PAC) significantly increased 2 days after $\mathrm{I} / \mathrm{R}$ and remained elevated at least 7 days after I/R. Treatment with benidipine significantly decreased I/R-induced elevation of the PAC. I/R-induced markers of fibrosis in hearts also reduced in benidipine. These results suggest that the administration of benidipine reduces myocardial infarct size as well as systemic oxidative stress after I/R. These phenomena are partially linked to reduced plasma aldosterone levels. Hypertension Research (2012) 35, 287-294; doi:10.1038/hr.2011.183; published online 24 November 2011
\end{abstract}

Keywords: aldosterone; calcium channel blocker; ischemia reperfusion; oxidative stress

\section{INTRODUCTION}

Early recanalization of an occluded coronary artery in acute myocardial infarction (MI) reduces the extent of myocardial necrosis, improves prognosis and preserves the function of the left ventricle (LV). ${ }^{1}$ On the other hand, opening an occluded coronary artery can sometimes induce myocardial ischemia reperfusion (I/R) injury. ${ }^{2}$ A growing body of evidence demonstrates that reperfusion after ischemia results in additional cell death and enhances infarct size, called I/R injury. ${ }^{3}$ One major component of I/R injury mediating the development and progression of postischemic myocardial damage is oxidative stress, which leads to cardiomyocytes' apoptosis. ${ }^{4}$

It is important to block the renin-angiotensin-aldosterone pathway after MI. Indeed, aldosterone might be essential to the pathophysiology of cardiovascular injury. ${ }^{5}$ Using a mineralocorticoid receptor (MR) antagonist in addition to standard therapy also appears to reduce clinical mortality, ${ }^{6}$ which suggests that MR activation may have a role in $\mathrm{MI}$ and heart failure. MR blockade is associated with decreased fibrosis, vascular inflammation, reduced oxidative stress and I/R injury, ${ }^{7}$ and endothelial dysfunction. ${ }^{8}$
Accordingly, aldosterone must be an important risk factor for cardiovascular diseases.

Benidipine, a long-acting dihydropyridine calcium channel blocker, is used widely to treat hypertension and ischemic heart disease., ${ }^{9,10}$ Benidipine evokes the vasodilatation of coronary and peripheral arteries by inhibiting calcium influx via L-type calcium channels. Benidipine also has the effect to block T- and N-type calcium channels. This unique calcium channel blocker exhibits additional pharmacological effects, such as limiting infarct size via bradykinin and nitric oxide (NO)dependent mechanisms in the canine heart,${ }^{11}$ and reducing the production of hydroxyl radicals in rabbits. ${ }^{12}$ Benidipine also inhibits the production of aldosterone in human adrenocortical cells through the suppression of T-type calcium channels. ${ }^{13}$ However, it remains unknown whether this calcium channel blocker attenuates myocardial $\mathrm{I} / \mathrm{R}$ injuries and aldosterone production in mice.

In this study, we demonstrated that benidipine would reduce MI size following $\mathrm{I} / \mathrm{R}$ in mice. We also showed that benidipine decreased I/R-induced oxidative stress through mechanisms partially involving downregulation in plasma aldosterone concentration (PAC). 


\section{METHODS}

An expanded Methods section is available in the Supplementary Information.

\section{Experimental protocol}

The experiments were approved by the Institutional and Governmental Animal Research Committees and were conducted in accordance with the Guide for the Care and Use of Laboratory Animals in Kanazawa University that strictly conforms to Guide for the Care and Use of Laboratory, published by the US NIH (National Institutes of Health; publication no. 85-23, revised 1996).

\section{I/R and administration of drugs}

The method for inducing myocardial I/R in mice has been described. ${ }^{14}$. Male C57BL/6J mice (BW $24.5 \pm 1.86 \mathrm{~g}, 8-12$ weeks of age, Charles River Laboratories, Yokohama, Japan) were subjected to $\mathrm{I} / \mathrm{R}$ or sham operation. Sham and $\mathrm{I} / \mathrm{R}+\mathrm{V}$ (vehicle) groups were orally administered $0.3 \%$ sodium carboxymethyl cellulose ${ }^{15}$ by gavage daily from 7 days before the operation to 14 days after it. In the I/ $\mathrm{R}+$ Beni groups, a $3 \mathrm{mg} \mathrm{kg}^{-1}$ dose of benidipine (Kyowa Kirin, Tokyo, Japan) dissolved by carboxymethyl cellulose was administered similarly and the same surgical procedures of I/R were followed. Nifedipine $\left(3 \mathrm{mg} \mathrm{kg}^{-1}\right)$ was orally administered daily from 7 days before operation in mice. Eplenerone (200 $\mathrm{mg} \mathrm{kg}^{-1}$ ) was administered daily from just before operation of I/R to 7 days after operation. In I/R+Beni+Aldo groups, mice received a continuous infusion of aldosterone by micro pump at a dose of $8 \mu \mathrm{gkg}^{-1}$ per day for 4 days. Previous reports $^{16-19}$ and preliminary experiments indicated that this dose of these drugs does not reduce blood pressure in C57BL/6J mice (Supplementary Tables 1 and 2).

\section{Measurement of PAC, plasma renin activity}

Then blood samples were collected from the LV apex in before operation and $24 \mathrm{~h}, 2,4$ and 7 days after operation, and PAC was measured by radioimmunoassay (Coat-a-count; DPC, Mitsubishi, Tokyo, Japan).$^{20}$ Plasma renin activity in $24 \mathrm{~h}$ after operation was measured by plasma renin activity kit (TFB, Tokyo, Japan). ${ }^{21}$

\section{Hemodynamic measurements}

Systolic blood pressure, mean blood pressure and heart rate were measured in unanesthetized mice using a computerized tail-cuff system ${ }^{22}$ (BP-98A system; Softron, Tokyo, Japan). The animals were placed in restraining units mounted on a warmed $\left(36-37^{\circ} \mathrm{C}\right)$ surface (THC-2; Softron), and their tails were passed through a cuff. Each session included $>10$ tail-cuff measurements.

\section{Determination of myocardial apoptosis}

Myocardial apoptosis was determined by TdT-mediated dUTP nick end labeling (TUNEL) staining using an in situ apoptosis detection kit (Takara, Shiga, Japan) $24 \mathrm{~h}$ after operating. The positively stained nuclei were counted under $\times 200$ magnification and the ratio of positively stained apoptotic cells to all cells was evaluated.

\section{Assessment of myocardial oxidative stress}

Myocardial oxidative stress was assessed by an immunohistochemical method using the mouse ImmunoCruz staining system (sc-2050; Santa Cruz Biotechnology, CA, USA) for 8-hydroxy-2'-deoxyguanosine (8-OHdG $)^{23} 24 \mathrm{~h}$ after the operation.

\section{Urine collection and assessment of systemic oxidative stress}

The urine samples, collected using metabolic cages for mice (Shinano, Tokyo, Japan) in before operation and $24 \mathrm{~h}, 2,4,7$ and 14 days after operation, were stored at $-80^{\circ} \mathrm{C}$ until use. We performed an enzyme-linked immunosorbent assay to detect urinary 8-OHdG using the New 8-OHdG Check enzyme-linked immunosorbent assay Kit (Japan Institute for the Control of Aging, Shizuoka, Japan).

\section{Isolation of mRNA and quantitative real-time PCR}

Total RNA was isolated using RNeasy (Qiagen, Valencia, CA, USA) from heart according to the protocol of the manufacturer. Total RNA $(1 \mu \mathrm{g})$ was used to generate cDNA using Rever Tra Ace (Toyobo, Osaka, Japan) according to the protocol of the manufacturer. Real-time Quantitative PCR analysis was perfomed using the ABI PRISM 7300 sequence detection system (Applied Biosystems, Foster, CA, USA). The following primers and TaqMan probes (Applied Biosystems) were used: interleukin 6 (Il6 Mm99999064_m1), tumor necrosis factor (Tnf, ID\# Mm00443258_m1), collagen, type I, $\alpha 1$ (Collal, ID\# Mm00801 666_g1), $\alpha$ smooth muscle actin (Acta2, ID\# Mm01546133_m1), gp91phox (cybb, ID\# Mm00432775_m1), p22phox (cyba, ID\# Mm00514478_m1), p47phox (ncf1, ID\# Mm00447921_m1), p67phox(ncf2, ID\# Mm00726636_s1) and MR(nr3c2, ID\# Mm01241596_m1). Glyceraldehyde-3-phsphate dehydrogenase (Gapdh, ID\# Mm99999915_g1) were used as an endogenous control.

Table 1 Hemodynamic parameters following I/R

\begin{tabular}{|c|c|c|c|c|c|c|}
\hline $\begin{array}{l}\text { Group } \\
\text { (Before operation) }\end{array}$ & Day 0 & Day 1 & Day 2 & Day 4 & Day 7 & Day 14 \\
\hline \multicolumn{7}{|c|}{ Heart rate (beats min $^{-1}$ ) } \\
\hline Sham & $564 \pm 21$ & $555 \pm 15$ & $562 \pm 18$ & $606 \pm 35$ & $570 \pm 28$ & $588 \pm 24$ \\
\hline $\mathrm{l} / \mathrm{R}+\mathrm{V}$ & $564 \pm 21$ & $588 \pm 27$ & $569 \pm 15$ & $596 \pm 13$ & $586 \pm 21$ & $595 \pm 19$ \\
\hline I/R+Beni & $622 \pm 14$ & $609 \pm 14$ & $554 \pm 17$ & $574 \pm 21$ & $600 \pm 17$ & $590 \pm 22$ \\
\hline \multicolumn{7}{|c|}{ Systolic blood pressure $(\mathrm{mm} \mathrm{Hg})$} \\
\hline Sham & $114 \pm 3$ & $103 \pm 2$ & $107 \pm 2$ & $110 \pm 2$ & $106 \pm 4$ & $106 \pm 3$ \\
\hline $\mathrm{I} / \mathrm{R}+\mathrm{V}$ & $114 \pm 3$ & $98 \pm 3$ & $101 \pm 2$ & $104 \pm 2^{*}$ & $101 \pm 3$ & $104 \pm 3$ \\
\hline I/R+Beni & $112 \pm 2$ & $96 \pm 3$ & $101 \pm 3$ & $102 \pm 2^{* *}$ & $102 \pm 4$ & $102 \pm 3$ \\
\hline \multicolumn{7}{|c|}{ Mean blood pressure ( $\mathrm{mm} \mathrm{Hg}$ ) } \\
\hline Sham & $79 \pm 2$ & $75 \pm 1$ & $78 \pm 1$ & $81 \pm 2$ & $76 \pm 4$ & $76 \pm 3$ \\
\hline $\mathrm{I} / \mathrm{R}+\mathrm{V}$ & $79 \pm 2$ & $71 \pm 2$ & $69 \pm 2^{* *}$ & $73 \pm 3^{*}$ & $73 \pm 3$ & $74 \pm 4$ \\
\hline I/R+Beni & $81 \pm 3$ & $70 \pm 4$ & $70 \pm 3 *$ & $72 \pm 2 *$ & $75 \pm 4$ & $71 \pm 3$ \\
\hline \multicolumn{7}{|l|}{ Urine volume $(\mathrm{ml})$} \\
\hline Sham & $0.96 \pm 0.11$ & $0.76 \pm 0.10$ & $0.86 \pm 0.10$ & $1.06 \pm 0.14$ & $1.04 \pm 0.08$ & $0.92 \pm 0.09$ \\
\hline $\mathrm{I} / \mathrm{R}+\mathrm{V}$ & $0.96 \pm 0.11$ & $0.67 \pm 0.11$ & $0.78 \pm 0.10$ & $1.02 \pm 0.10$ & $0.91 \pm 0.09$ & $0.91 \pm 0.11$ \\
\hline I/R+Beni & $0.92 \pm 0.09$ & $0.68 \pm 0.06$ & $0.83 \pm 0.09$ & $0.81 \pm 0.06$ & $0.83 \pm 0.09$ & $0.89 \pm 0.12$ \\
\hline
\end{tabular}

Abbreviation: $I / R$, ischemia reperfusion.

Values are expressed as mean \pm s.e.m. ( $N=7-9 /$ group).

Values are expressed as mean \pm s.e.m. $(N=7-9$ /group).
${ }^{*} P<0.05$ vs. sham group, ${ }^{*} P<0.01$ vs. sham group. 


\section{Statistical analysis}

The values, represented as the mean \pm s.e. for each group of mice, were assessed by analysis of variance with a subsequent Bonferroni's post hoc test for multiple comparisons. The significance of differences between two groups was determined using the Student's $t$-test. Associations of urinary 8-OHdG and fractional shortening were assessed by Pearson's correlation coefficient. Statistical significance was established at a value of $P<0.05$. All statistical analyses were performed using StatView software (SAS Institute, Cary, NC, USA).

\section{RESULTS}

\section{Hemodynamic parameters}

Before the operation, the administration of benidipine did not affect heart rate, systolic/mean blood pressure and urine volume (Table 1). a

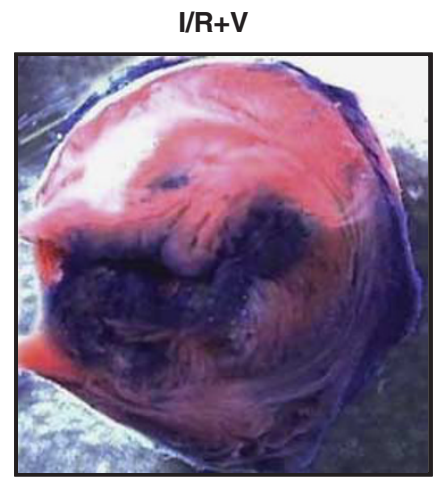

b

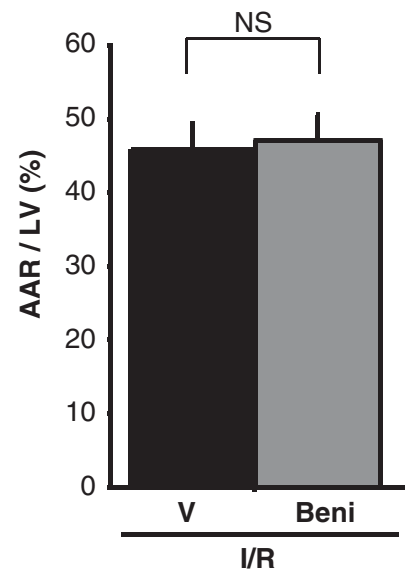

d

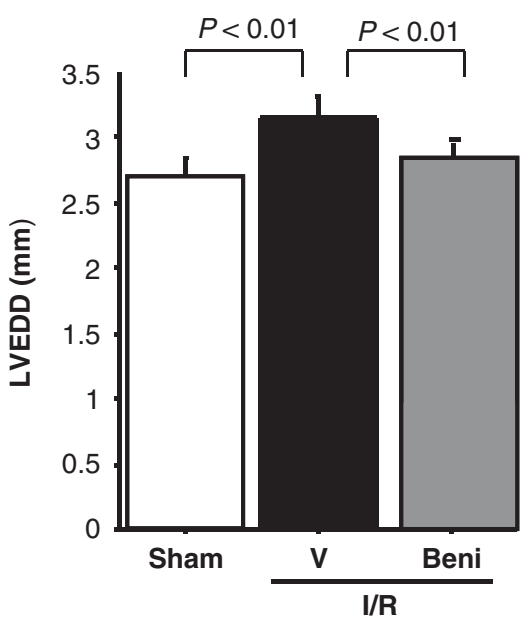

I/R+Beni

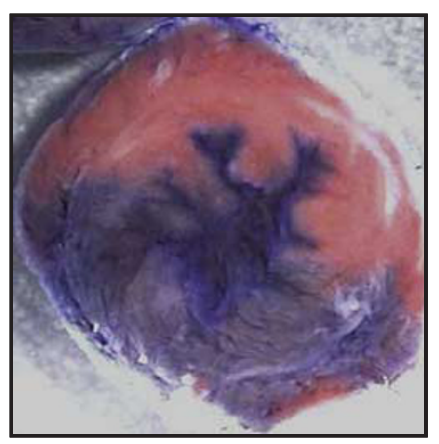

C

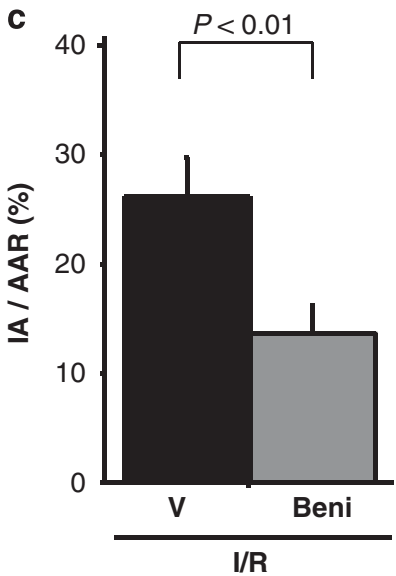

e
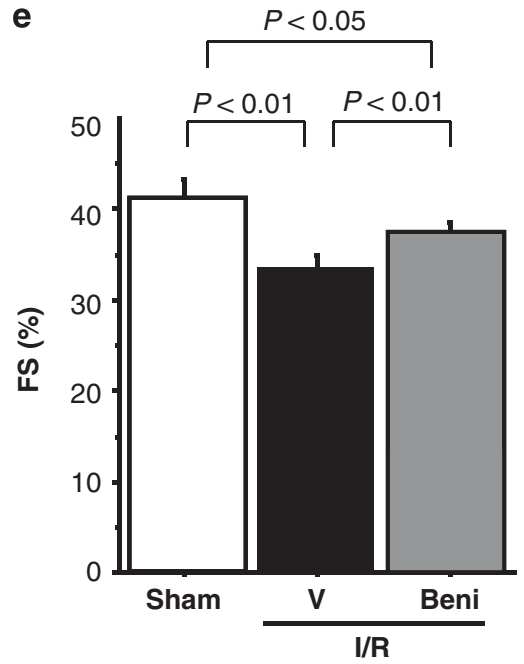

Figure 1 (a) Representative examples of myocardial infarction stained with Evans blue (EB)/triphenyl tetrazolium chloride (TTC) $24 \mathrm{~h}$ after I/R. Benidipine decreases infarct size. EB-stained areas (purple staining) indicate nonischemic regions; TTC-stained areas (red staining) indicate ischemic but viable tissue; EB/ TTC-stained negative (white) areas indicate myocardium infarcts. (b) Area at risk (AAR) and infarct area (IA) of the $I / R+V$ and $I / R+B e n i$ groups. There was no significant difference in the AAR between the two groups. Infarct size was significantly reduced in the I/R+Beni group compared with that of the $\mathrm{I} / \mathrm{R}+\mathrm{V}$ group (c). $\mathrm{LV}$, left ventricular area. $N=4$ per group. Data are expressed as mean \pm s.e.m. (d), (e) Effect of benidipine on myocardial function 7 days after ischemic reperfusion as determined by echocardiography. LVEDD, left ventricular end-diastolic diameter; FS, fractional shortening. $N=10$ per group. Data are expressed as mean \pm s.e.m. 
The heart rate and urine volume did not differ significantly at each time point among the three groups throughout the protocol. In mice subjected to I/R, the systolic and mean blood pressures 2 and 4 days after operation were significantly lower than those of the sham groups, with or without benidipine. These results indicate that there were no significant changes in hemodynamic parameters during the oral administration of benidipine.

\section{Effect of benidipine on myocardial infarct size and LV function after $\mathbf{I} / \mathbf{R}$}

To examine the effects of benidipine on cardioprotection during $\mathrm{I} / \mathrm{R}$ injury, we applied $30 \mathrm{~min}$ of ischemia and a subsequent $24 \mathrm{~h}$ of reperfusion to $\mathrm{I} / \mathrm{R}$ mice. The mean area at risk (AAR) in the $\mathrm{LV}$ area was no different with or without benidipine (Figure $1 \mathrm{~b}$ ). I/R significantly increased IA/AAR after $24 \mathrm{~h}$ of reperfusion compared with the sham mice, but the administration of benidipine significantly attenuated I/R-induced increases in IA/AAR (Figures $1 \mathrm{a}$ and $\mathrm{c}$ ). To examine the effect of L-type calcium channel blocker on I/R injury, nifedipine, a pure L-type calcium channel blocker, was administrated. The administration of nifedipine did not affect the heart rate and systolic and mean blood pressures after I/R. IA/AAR after $24 \mathrm{~h}$ of reperfusion was similar in $\mathrm{I} / \mathrm{R}+\mathrm{Nife}$ compared with $\mathrm{IR}+\mathrm{V}$ groups (Supplementary Figure 1).

Echocardiographic measurements showed that I/R resulted in LV dilatation and decreased percentage fractional shortening, compared with sham mice. The administration of benidipine significantly attenuated I/R-induced increases in left ventricular end-diastolic diameter (Figure 1d) as well as decreases in percentage fractional shortening $2.84 \pm 0.02$ vs. $3.15 \pm 0.07 \mathrm{~mm}, P<0.01,37.6 \pm 0.5$ vs. $33.3 \pm 0.8 \%, P<0.01$, respectively; Figure 1e). Taken together, these results suggest that benidipine, not nifedipine, attenuates I/R-induced myocardial infarct size and LV dysfunction.

a

I/R

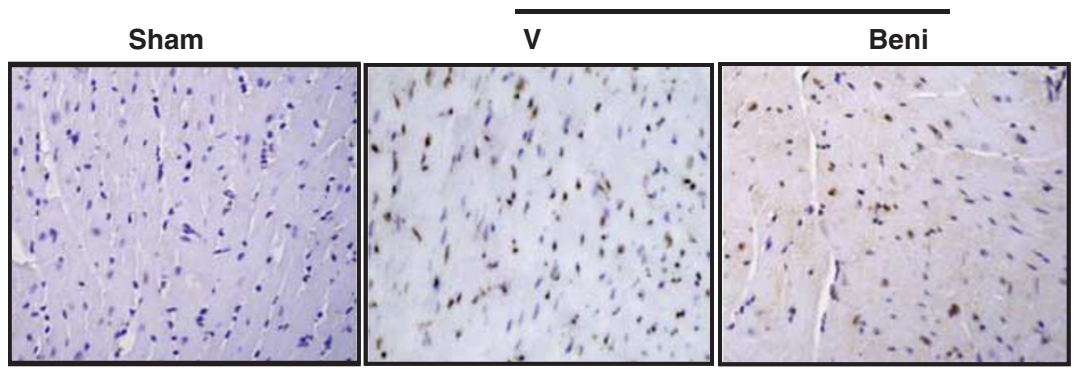

b

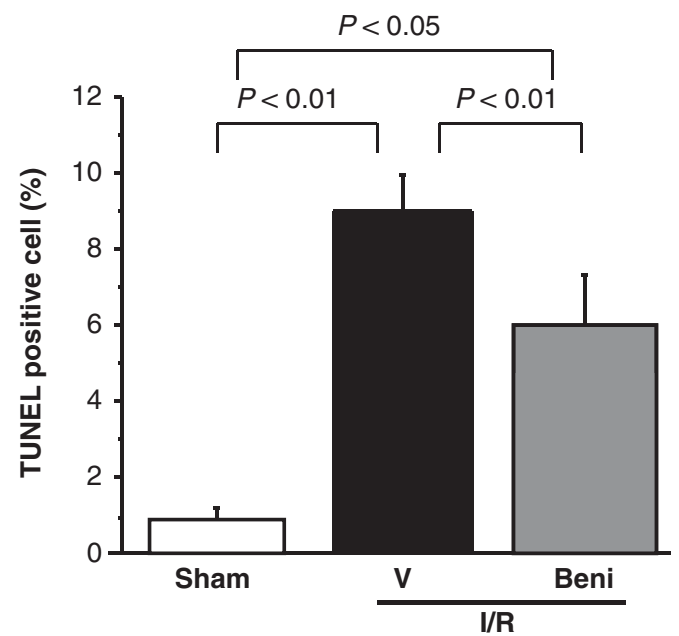

C

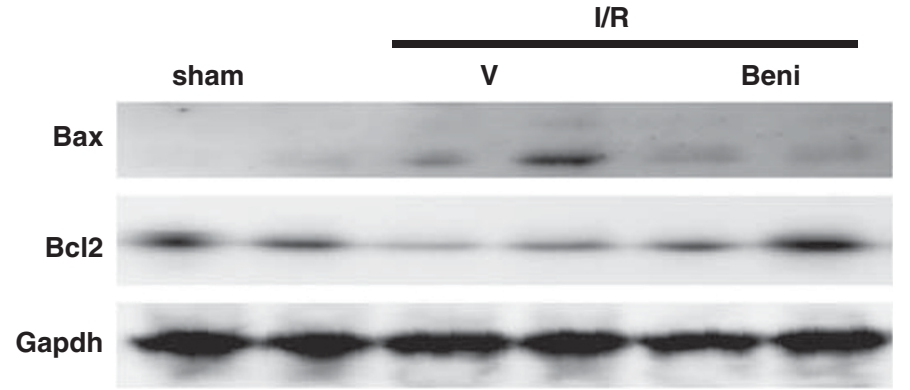

Figure 2 (a) Representative examples of myocardial apoptotic cells by TdT-mediated dUTP nick end labeling (TUNEL) staining of the area at risk $24 \mathrm{~h}$ after operation. There were fewer TUNEL-positive cells (dark brown) in the $I / R+B e n i$ group than in the $I / R+V$ group. There was a significant difference in the TUNEL-positive cells among the three groups (b). Myocardial apoptosis was significantly lower in the I/R+Beni group than the I/R+V group. (c) Immunoblots with anti-bax and anti-bcl2 antibodies are shown. $N=6-7$ per group. Data are expressed as mean \pm s.e.m. 


\section{Benidipine attenuates apoptosis following $I / R$}

$\mathrm{I} / \mathrm{R}$ injury is associated with increased apoptosis in the myocardium. To determine the extent of apoptosis, TUNEL-positive nuclei were counted among 5000 nuclei in the AAR of each mouse. The number of TUNELpositive myocytes was significantly higher in $\mathrm{I} / \mathrm{R}+\mathrm{V}$ groups than in sham mice ( $9.0 \pm 0.9 \%$ vs. $1.0 \pm 0.3 \%, P<0.0001$; Figures $2 \mathrm{a}$ and $\mathrm{b})$, suggesting that apoptosis is enhanced during cardiac $I / R$. The number of TUNEL-positive myocytes was also induced in $\mathrm{I} / \mathrm{R}+\mathrm{Beni}$ groups, but I/R-induced TUNEL-positive myocyte was significantly lower than in $\mathrm{I} / \mathrm{R}+\mathrm{V}$ groups $(6.0 \pm 1.3$ vs. $9.0 \pm 0.9 \%, P<0.05)$. Protein levels of total bax were increased and protein levels of bcl-2 were decreased in $\mathrm{I} / \mathrm{R}+\mathrm{V}$ groups (Figure 2c). I/R-induced changes of bax and bcl-2 were not observed in I/R+Beni groups. Furthermore, the level of endothelial NO synthase phosphorylation was increased after I/R. I/R-induced endothelial NO synthase phosphorylation was higher in $\mathrm{I} / \mathrm{R}+\mathrm{Beni}$ than in $\mathrm{I} / \mathrm{R}+\mathrm{V}$ (Supplementary Figure 2). These results suggest that benidipine inhibits cardiac myocyte apoptosis induced by I/R.

\section{Myocardial oxidative stress after $I / R$}

To examine oxidative stress after $\mathrm{I} / \mathrm{R}$ in hearts, we evaluated the myocardial levels of $8-\mathrm{OHdG}$, a sensitive indicator of oxidative DNA damage. As shown in Figure 3, I/R significantly increased 8-OHdG expression in the AAR $(12.4 \pm 1.3$ vs. $2.7 \pm 0.4 \%, P<0.0001)$. The administration of benidipine attenuated this response $(6.7 \pm 0.4$ vs. $12.4 \pm 1.3 \%, P<0.05)$. The expression of gp91phox, NAD(P)H oxidase, mRNA was decreased in $\mathrm{I} / \mathrm{R}+\mathrm{Beni}$ compared with $\mathrm{I} / \mathrm{R}+\mathrm{V}$ (Supplementary Figure 3). These results suggest that benidipine attenuates cardiac oxidative stress after $\mathrm{I} / \mathrm{R}$.

\section{Aldosterone plasma levels after I/R}

Plasma aldosterone levels were markedly elevated after I/R, with a peak at 2 days that gradually declined thereafter but remained elevated for at least 7 days (Figure 4a). Plasma renin activities were significantly increased in both $\mathrm{I} / \mathrm{R}+\mathrm{V}$ and $\mathrm{I} / \mathrm{R}+\mathrm{Beni}$ groups compared with sham (Supplementary Table 3). Administration of benidipine in this model was not associated with an increase in serum renin activities. I/R was not affected the levels of serum potassium. Interestingly, the administration of benidipine significantly inhibited this response 2 and 4 days after I/R. The administration of nifedipine did not affect aldosterone levels after I/R (Figure $4 \mathrm{~b}$ ). These results suggest that benidipine inhibits aldosterone production in response to $\mathrm{I} / \mathrm{R}$.

\section{Systemic oxidative stress and cardiac remodeling after I/R}

We estimated the ratio of urinary 8-OHdG to creatinine, a marker of systemic oxidative DNA damage induced by reactive oxygen species. Urinary $8-\mathrm{OHdG} /$ creatinine levels increased significantly in mice subjected to $\mathrm{I} / \mathrm{R}$ compared with sham mice after operation (days 1 , 2, 4 and 7). Benidipine significantly attenuated this response (Figure 5a), as did the administration of eplerenone, a selective mineral corticoid receptor antagonist (Figure 5b). Furthermore, continuous infusion of aldosterone abolished the benidipine's inhibition of I/R-induced urinary $8-\mathrm{OHdG} /$ creatinine levels. These results suggest that benidipine attenuates cardiac and systemic oxidative stress after I/R through aldosterone production. We also examined the effect of benidipine administration for cardiac inflammation and remodeling after I/R. We isolated heart tissues 4 days after I/R for quantitative real-time PCR. The mRNA expression level of MR was no changes in

a I/R

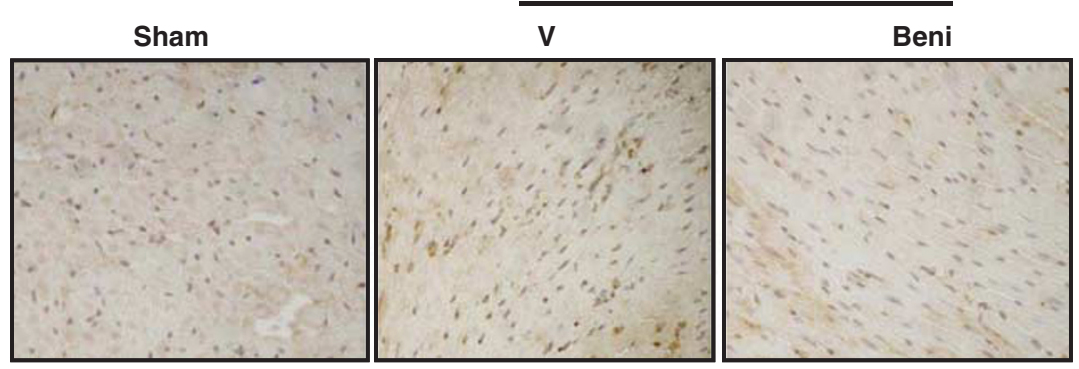

b

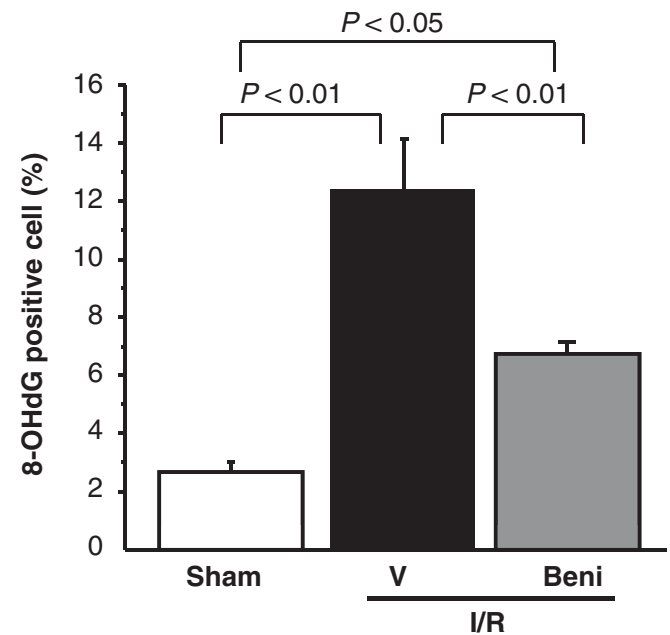

Figure 3 (a) Representative examples of myocardial 8-hydroxydeoxyguanosine (8-OHdG) staining by an immunohistochemical method at the area at risk $24 \mathrm{~h}$ after operation. There were fewer 8-OHdG-positive cells (brown) in the I/R+Beni group than in the I/R+V group. There was a significant difference in the

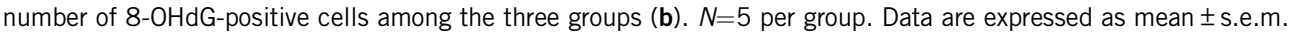




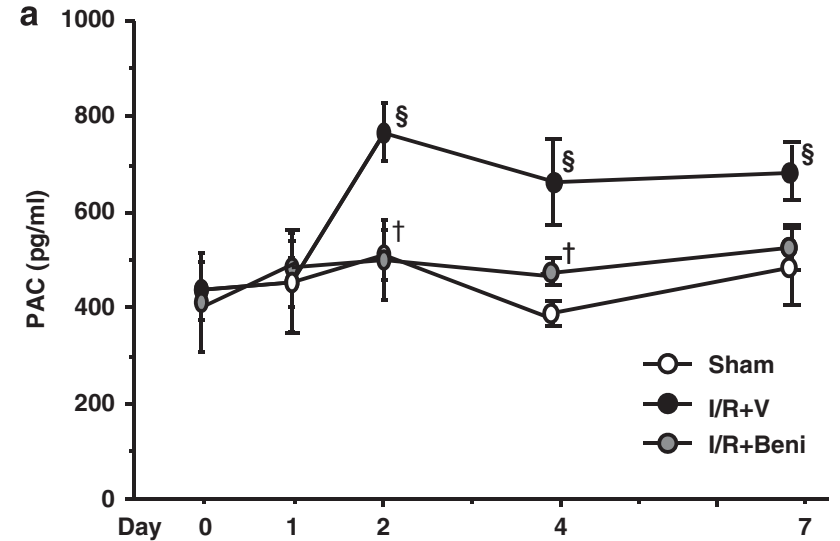

b

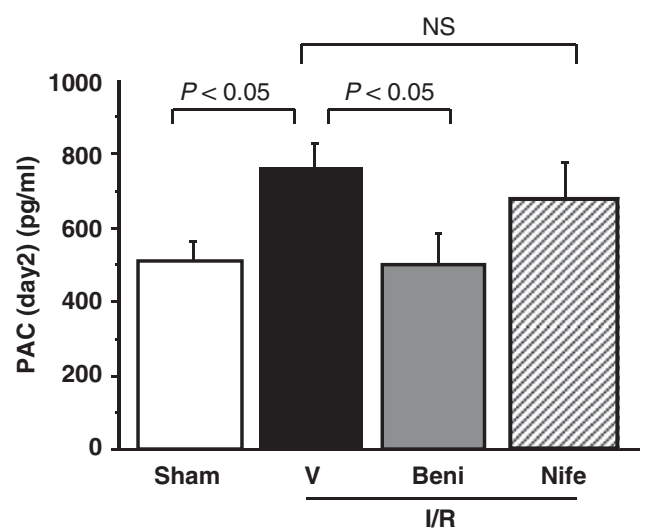

Figure 4 (a) Effect of benidipine on plasma aldosterone concentration (PAC), from before I/R to 7 days after $\mathrm{I} / \mathrm{R}$ ( $N=5$ per group). (b) PAC in the I/R group that was administered nifedipine $\left(3 \mathrm{mg} \mathrm{kg}^{-1}\right.$ per day from 7 days before) compared with three other groups on day 2 . Nifedipine did not significantly inhibit the elevation of PAC-induced I/R $(N=5)$. Data are expressed as mean \pm s.e.m. ${ }^{\S} P<0.05$ vs. sham group, ${ }^{\dagger} P<0.05$ vs. $I / R+V$ group.

both $\mathrm{I} / \mathrm{R}+\mathrm{V}$ and $\mathrm{I} / \mathrm{R}+\mathrm{Beni}$ compared with sham groups (Supplementary Figure 4). Multiple markers of inflammation and fibrotic remodeling were elevated in I/R compared with sham. I/R-induced mRNA upregulation of interleukin 6 , tumor necrosis factor- $\alpha$, Collal and Acta2 were decreased in the $I / R+B e n i$ groups (Figures $5 c-f$ ). In aldosterone infusion mice, mRNA upregulation of only Colla1 was significantly elevated. Continuous infusion of aldosterone partially reduced the beneficial effect of benidipine. These results indicate that administration of benidipine inhibited I/R-induced cardiac inflammation and fibrotic remodeling, partially through the production of aldosterone.

\section{DISCUSSION}

In this study, the long-acting dihydropyridine calcium channel blocker benidipine, when administered at a dose that did not decrease blood pressure, reduced the myocardial infarct size, apoptosis of cardiac myocytes and myocardial $8-\mathrm{OHdG}$ in I/R. These results suggest that the cardioprotective effects of benidipine may be independent of its anti-hypertensive effects.

This study demonstrated the following major findings. First, benidipine reduces infarct size and may be improve cardiac dysfunction following I/R. Second, benidipine reduces the PAC that increases due to $I / R$. Finally, benidipine inhibits $I / R$-induced increases in urinary 8 -OHdG and cardiac inflammatory responses and fibrotic remodeling. Thus, these results suggest that benidipine may be a useful cardioprotective agent against oxidative stress and could potentially serve as a therapeutic strategy for I/R injury.

It was previously demonstrated that benidipine limits $I / R$ injury in vivo. In canine $\mathrm{I} / \mathrm{R}$ hearts, benidipine increased the $\mathrm{NO}$ and bradykinin levels of coronary venous blood and limited infarct size. ${ }^{11}$ Wang et al. ${ }^{12}$ reported that benidipine reduced myocardial infarct size by reducing hydroxyl radicals and inducing the production of NO in rabbits. Moreover, benidipine has been shown to reduce postischemic myocardial apoptosis by inhibiting caspase-3 activation. ${ }^{24}$ In this study, we demonstrated that the oral administration of benidipine reduces myocardial infarct size. Expression of bax protein was increased and bcl 2 protein was decreased in hearts after I/R. These changes in apoptotic regulating proteins did not occur by administration of benidipine. Consequently, the number of TUNELpositive cells was smaller in benidipine treatment. We also demonstrated that benidipine increased endothelial NO synthase activity in hearts. On the basis of our results, we suggest that the reduction of hydroxyl radicals may have one of the important key roles in the cardioprotective effect of benidipine.

Benidipine treatment $\left(3 \mathrm{mg} \mathrm{kg}^{-1}\right.$ per day) did not affect to blood pressure and heart rate. Even after I/R, these parameters showed no difference with or without benidipine. Consistent with previous work, ${ }^{16}$ these results suggest that the cardioprotective effect of benidipine is likely to be independent of blood pressure, because the dose of benidipine used in this study did not significantly affect systemic blood pressure.

The generation of free radicals and oxidants is associated with the exacerbation of reperfusion injury in myocardial I/R. $.^{25} 8-\mathrm{OHdG}$ is a sensitive and integral marker of oxidative damage to DNA. Cordis et al. ${ }^{26}$ reported that 8 -OHdG is formed at the onset of reperfusion in the myocardium. Benidipine treatment reduces the level of hydroxyl radicals in reperfused myocardium in rabbit. ${ }^{11}$ In this study, 8-OHdG accumulated in mouse hearts $24 \mathrm{~h}$ after reperfusion, but benidipine treatment reduced $8-\mathrm{OHdG}$ levels. Benidipine also suppressed increases of gp91phox, one of the important components of $\mathrm{NAD}(\mathrm{P}) \mathrm{H}$ oxidase, after I/R. In addition, urinary 8 -OHdG, a marker of systemic oxidative stress, was elevated in both the early ( 1 and 2 days after reperfusion) and late phases (4 and 7 days after reperfusion), and benidipine reduced urinary $8-\mathrm{OHdG}$ levels in both phases. We suggest that reduction of myocardial oxidative stress is one of the mechanism through which benidipine decreases infarct size, and that myocardial oxidative stress reflect systemic oxidative stress in the early phase after reperfusion.

Aldosterone, an antidiuretic hormone, has an important role in regulating electrolyte composition by promoting sodium retention and potassium excretion ${ }^{27}$ and MR antagonist regressed hypertrophy, fibrosis and diastolic dysfunction in human and experimental hypertensive heart disease. ${ }^{28,29}$ An excess of mineralocorticoid causes cardiac hypertrophy, fibrosis and diastolic dysfunction. ${ }^{30,31}$ In this study, we found for the first time that the plasma concentration of aldosterone increased after I/R partially through the upregulation of plasma renin activity and that benidipine treatment effectively inhibited this response in vivo. Aldosterone is produced mainly by the adrenal cortex in response to increased potassium or angiotensin II. ${ }^{32}$ Benidipine inhibits angiotensin II-induced aldosterone production by regulating T-type calcium channels. ${ }^{13}$ The synthesis of aldosterone requires the aldosterone synthetase CYP11B2. ${ }^{33}$ Several reports have shown that there isCYP11B2 mRNA expression and activity in the heart and vessel walls; ${ }^{34}$ however, results regarding the synthesis of aldosterone in the heart and peripheral vasculature are 
a

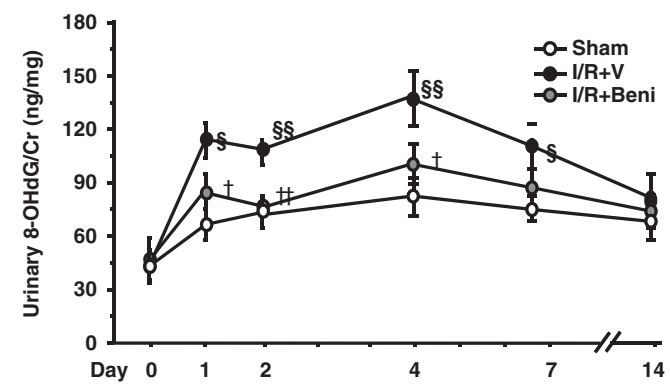

b

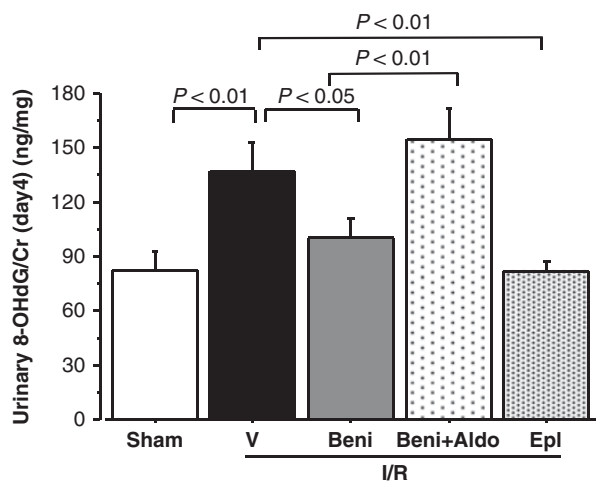

C
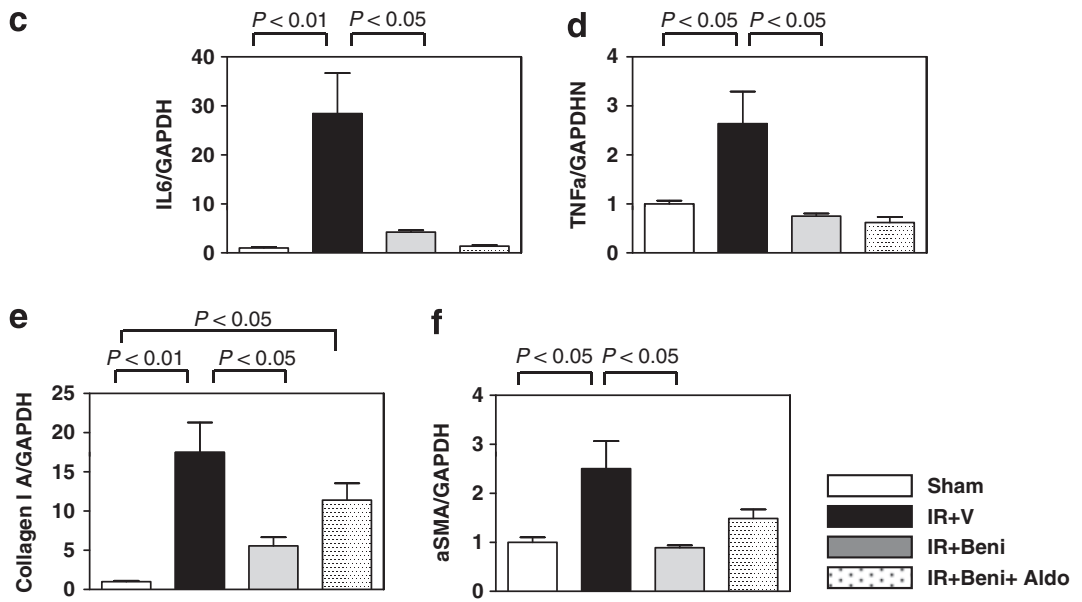

Figure 5 (a) Effect of benidipine on systemic oxidative stress before I/R to 14 days after I/R as determined by urinary 8-OHdG/creatinine $(\mathrm{Cr})$ levels $(N=9-$ 10 per group). ${ }^{\$} P<0.05$ vs. the sham group, ${ }^{\$} P<0.05$ vs. sham group, ${ }^{\dagger} P<0.05$ vs. $\mathrm{I} / \mathrm{R}+\mathrm{V}$ group, ${ }^{\dagger \dagger} P<0.01$ vs. $\mathrm{I} / \mathrm{R}+\mathrm{V}$ group. (b) The urinary $8-\mathrm{OHdG} / \mathrm{Cr}$ level on day 4. Eplerenone significantly inhibited the elevation of I/R-induced urinary $8-\mathrm{OHdG} / \mathrm{Cr}$ levels $(N=10)$. Continuous infusion of aldosterone canceled the suppression of benidipine in I/R-induced urinary $8-\mathrm{OHdG} / \mathrm{Cr}$ levels. Inflammatory cytokines and fibrotic markers expression. Myocardial mRNA levels of interleukin 6 (c), tumor necrosis factor- $\alpha$ (d), Collagen la (e), and $\alpha$ SMA (f). Data are expressed as mean \pm s.e.m.

contradictory. ${ }^{35}$ We tried to examine the mRNA expression levels of CYP11B2 in the heart, aorta and kidney using quantitative real-time PCR, but we could not detect the expression of CYP11B2 in any tissues (data not shown). Furthermore, we found that the administration of nifedipine, a pure L-type calcium channel blocker, did not inhibit I/R-induced aldosterone production. On the basis of these results, we speculate that benidipine inhibits aldosterone synthesis in the adrenal gland by blocking T-type calcium channels. At present, however, this hypothesis remains to be tested.

Oxidative stress and inflammation lead to increased cardiovascular morbidity and mortality. ${ }^{36}$ Much evidence indicates that aldosterone and/or MR activation result in oxidative stress and vascular inflammation. Aldosterone itself enhances $\mathrm{NAD}(\mathrm{P}) \mathrm{H}$ oxidase activation, ${ }^{37}$ a major source of reactive oxygen species, and the MR blocker inhibits angiotensin II-induced activation of $\mathrm{NAD}(\mathrm{P}) \mathrm{H}$ oxidase activity and myocardial interstitial fibrosis after MI. This study demonstrates the I/R-induced enhancement of urinary 8 -OHdG in the late phase and elevation of PAC. Interestingly, the elevation in urinary $8-\mathrm{OHdG}$ was reduced by both benidipine and eplerenone in the late phase after reperfusion. Continuous infusion of aldosterone was abolished beneficial effects of benidipine after I/R. These results suggest that $\mathrm{MR}$ regulates $\mathrm{I} / \mathrm{R}$-induced increases in 8 -OHdG. Benidipine also reduced I/R-induced inflammatory and fibrotic responses in hearts. These effects partially canceled by continuous infusion of aldosterone. In addition, benidipine also appears to have renoprotective and vascular endothelial protective effects. ${ }^{38}$ These results further suggest that the inhibition of aldosterone production and oxidative stresses contributes to the cardioprotective effect of benidipine and that 
benidipine provides a beneficial effect to multiple organs following cardiac I/R.

In conclusion, benidipine, a calcium channel blocker, reduces $I / R$ injury by inhibiting cardiac myocyte apoptosis, aldosterone production, oxidative stress and inflammation in mice. Our results suggest that benidipine, as a T-type calcium channel blocker, has additional benefits for cardiovascular disease beyond its anti-hypertensive effects. Thus, the use of benidipine is a potential therapeutic strategy for $\mathrm{I} / \mathrm{R}$ injury.

\section{CONFLICT OF INTEREST}

The authors declare no conflict of interest.

\section{ACKNOWLEDGEMENTS}

We thank Ayano Nomura for excellent technical support. This work was supported by a Grant-in-Aid for Young Scientists (B) awarded to SU.

1 Weaver WD, Simes RJ, Betriu A, Grines CL, Zijlstra F, Garcia E, Grinfeld L, Gibbons RJ, Ribeiro EE, DeWood MA, Ribichini F. Comparison of primary coronary angioplasty and intravenous thrombolytic therapy for acute myocardial infarction: a quantitative review. JAMA 1997; 278: 2093-2098.

2 Braunwald E, Kloner RA. Myocardial reperfusion: a double-edged sword? J Clin Invest 1985; 76: 1713-1719.

3 Yellon DM, Hausenloy DJ. Myocardial reperfusion injury. N Engl J Med 2007; 357: 1121-1135.

4 Lefer DJ, Granger DN. Oxidative stress and cardiac disease. Am J Med 2000; 109 . 315-323.

5 Rocha R, Stier Jr CT, Kifor I, Ochoa-Maya MR, Rennke HG, Williams GH, Adler GK. Aldosterone: a mediator of myocardial necrosis and renal arteriopathy. Endocrinology 2000; 141: 3871-3878.

6 Pitt B, Remme W, Zannad F, Neaton J, Martinez F, Roniker B, Bittman R, Hurley S, Kleiman J, Gatlin M. Eplerenone, a selective aldosterone blocker, in patients with left ventricular dysfunction after myocardial infarction. N Engl J Med 2003; 348. 1309-1321.

7 Schmidt K, Tissier R, Ghaleh B, Drogies T, Felix SB, Krieg T. Cardioprotective effects of mineralocorticoid receptor antagonists at reperfusion. Eur Heart J 2010; 31: 1655-1662.

8 Farquharson CA, Struthers AD. Spironolactone increases nitric oxide bioactivity, improves endothelial vasodilator dysfunction, and suppresses vascular angiotensin i/ angiotensin ii conversion in patients with chronic heart failure. Circulation 2000; 101 . 594-597.

9 Hoshide S, Kario K, Mitsuhashi T, Ikeda U, Shimada K. Is there any difference between intermediate-acting and long-acting calcium antagonists in diurnal blood pressure and autonomic nervous activity in hypertensive coronary artery disease patients? Hypertens Res 2000; 23: 7-14.

10 Ogihara T, Matsuzaki M, Matsuoka H, Shimamoto K, Shimada K, Rakugi H, Umemoto S, Kamiya A, Suzuki N, Kumagai H, Ohashi Y, Takishita S, Abe K, Saruta T. The combination therapy of hypertension to prevent cardiovascular events (cope) trial: Rationale and design. Hypertens Res 2005; 28: 331-338.

11 Asanuma H, Kitakaze M, Node K, Takashima S, Sakata Y, Asakura M, Sanada S, Shinozaki Y, Mori H, Tada M, Kuzuya T, Hori M. Benidipine, a long-acting ca channel blocker, limits infarct size via bradykinin- and no-dependent mechanisms in canine hearts. Cardiovasc Drugs Ther 2001; 15: 225-231.

12 Wang N, Minatoguchi S, Chen XH, Arai M, Uno Y, Lu C, Misao Y, Nagai H, Takemura G, Fujiwara $\mathrm{H}$. Benidipine reduces myocardial infarct size involving reduction of hydroxyl radicals and production of protein kinase c-dependent nitric oxide in rabbits. J Cardiovasc Pharmacol 2004; 43: 747-757.

13 Akizuki O, Inayoshi A, Kitayama T, Yao K, Shirakura S, Sasaki K, Kusaka H, Matsubara M. Blockade of t-type voltage-dependent ca2+ channels by benidipine, a dihydropyridine calcium channel blocker, inhibits aldosterone production in human adrenocortical cell line nci-h295r. Eur J Pharmacol 2008. 584: 424-434.

14 Takuwa N, Ohkura S, Takashima S, Ohtani K, Okamoto Y, Tanaka T, Hirano K, Usui S, Wang F, Du W, Yoshioka K, Banno Y, Sasaki M, Ichi I, Okamura M, Sugimoto N, Mizugishi K, Nakanuma Y, Ishii I, Takamura M, Kaneko S, Kojo S, Satouchi K, Mitumori K, Chun J, Takuwa Y. S1p3-mediated cardiac fibrosis in sphingosine kinase 1 transgenic mice involves reactive oxygen species. Cardiovasc Res 2010; 85: 484-493.
15 Yao K, Sato H, Ina Y, Suzuki K, Ohno T, Shirakura S. Renoprotective effects of benidipine in combination with angiotensin ii type 1 receptor blocker in hypertensive dahl rats. Hypertens Res 2003; 26: 635-641.

16 Yamashita T, Kawashima S, Ozaki M, Rikitake Y, Hirase T, Inoue N, Hirata K, Yokoyama M. A calcium channel blocker, benidipine, inhibits intimal thickening in the carotid artery of mice by increasing nitric oxide production. J Hypertens 2001; 19: 451-458.

17 Qin W, Rudolph AE, Bond BR, Rocha R, Blomme EA, Goellner JJ, Funder JW, McMahon EG. Transgenic model of aldosterone-driven cardiac hypertrophy and heart failure. Circ Res 2003; 93: 69-76.

18 Tsukuda K, Mogi M, Li JM, Iwanami J, Min LJ, Sakata A, Fujita T, Iwai M, Horiuchi M. Diabetes-associated cognitive impairment is improved by a calcium channel blocker, nifedipine. Hypertension 2008; 51: 528-533.

19 Brem AS, Morris DJ, Ge Y, Dworkin LD, Tolbert E, Gong R. Direct fibrogenic effects of aldosterone on normotensive kidney: An effect modified by 11 \{beta\}-hsd activity. Am J Physiol Renal Physiol 2010; 298: F1178-F1187.

20 Makhanova N, Lee G, Takahashi N, Sequeira Lopez ML, Gomez RA, Kim HS, Smithies O. Kidney function in mice lacking aldosterone. Am J Physiol Renal Physiol 2006; 290: F61-F69.

21 Yamamoto E, Kataoka K, Dong YF, Nakamura T, Fukuda M, Nako H, Ogawa H, KimMitsuyama S. Benidipine, a dihydropyridine I-type/t-type calcium channel blocker, affords additive benefits for prevention of cardiorenal injury in hypertensive rats. J Hypertens 2010; 28: 1321-1329.

22 Sabrane K, Kruse MN, Fabritz L, Zetsche B, Mitko D, Skryabin BV, Zwiener M, Baba HA, Yanagisawa M, Kuhn M. Vascular endothelium is critically involved in the hypotensive and hypovolemic actions of atrial natriuretic peptide. J Clin Invest 2005; 115: 1666-1674.

23 Roy D, Floyd RA, Liehr JG. Elevated 8-hydroxydeoxyguanosine levels in DNA of diethylstilbestrol-treated syrian hamsters: Covalent DNA damage by free radicals generated by redox cycling of diethylstilbestrol. Cancer Res 1991; 51: 3882-3885.

24 Liu HR, Gao F, Tao L, Yan WL, Gao E, Christopher TA, Lopez BL, Hu A, Ma XL. Antiapoptotic mechanisms of benidipine in the ischemic/reperfused heart. $\mathrm{Br} J$ Pharmacol 2004; 142: 627-634.

25 Matsubara M, Akizuki O, Ikeda J, Saeki K, Yao K, Sasaki K. Benidipine, an antihypertensive drug, inhibits reactive oxygen species production in polymorphonuclear leukocytes and oxidative stress in salt-loaded stroke-prone spontaneously hypertensive rats. Eur J Pharmacol 2008; 580: 201-213.

26 Cordis GA, Maulik G, Bagchi D, Riedel W, Das DK. Detection of oxidative DNA damage to ischemic reperfused rat hearts by 8-hydroxydeoxyguanosine formation. J Mol Cell Cardiol 1998; 30: 1939-1944.

27 Cohn JN, Colucci W. Cardiovascular effects of aldosterone and post-acute myocardial infarction pathophysiology. Am J Cardiol 2006; 97: 4F-12F.

28 Pitt B, Reichek N, Willenbrock R, Zannad F, Phillips RA, Roniker B, Kleiman J, Krause S, Burns D, Williams GH. Effects of eplerenone, enalapril, and eplerenone/enalapril in patients with essential hypertension and left ventricular hypertrophy: the 4e-left ventricular hypertrophy study. Circulation 2003; 108: 1831-1838.

29 Kuster GM, Kotlyar E, Rude MK, Siwik DA, Liao R, Colucci WS, Sam F. Mineralocorticoid receptor inhibition ameliorates the transition to myocardial failure and decreases oxidative stress and inflammation in mice with chronic pressure overload. Circulation 2005; 111: 420-427.

30 Brilla CG, Weber KT. Reactive and reparative myocardial fibrosis in arterial hypertension in the rat. Cardiovasc Res 1992; 26: 671-677.

31 Mohammed SF, Ohtani T, Korinek J, Lam CS, Larsen K, Simari RD, Valencik ML, Burnett Jr JC, Redfield MM. Mineralocorticoid accelerates transition to heart failure with preserved ejection fraction via "Nongenomic effects". Circulation 2010; 122 . 370-378.

32 Quinn SJ, Williams GH. Regulation of aldosterone secretion. Annu Rev Physiol 1988; 50: 409-426.

33 Denner K, Doehmer J, Bernhardt R. Cloning of cyp11b1 and cyp11b2 from normal human adrenal and their functional expression in cos-7 and v79 chinese hamster cells. Endocr Res 1995; 21: 443-448.

34 Takeda Y, Yoneda T, Demura M, Miyamori I, Mabuchi H. Cardiac aldosterone production in genetically hypertensive rats. Hypertension 2000; 36: 495-500.

35 Ye P, Kenyon CJ, MacKenzie SM, Jong AS, Miller C, Gray GA, Wallace A, Ryding AS, Mullins JJ, McBride MW, Graham D, Fraser R, Connell JM, Davies E. The aldosterone synthase (cyp11b2) and 11beta-hydroxylase (cyp11b1) genes are not expressed in the rat heart. Endocrinology 2005; 146: 5287-5293.

36 Griendling KK, Sorescu D, Ushio-Fukai M. Nad(p)h oxidase: Role in cardiovascular biology and disease. Circ Res 2000; 86: 494-501.

37 Johar S, Cave AC, Narayanapanicker A, Grieve DJ, Shah AM. Aldosterone mediates angiotensin ii-induced interstitial cardiac fibrosis via a nox2-containing nadph oxidase. FASEB J 2006; 20: 1546-1548.

38 Yao K, Sato $H$, Sonoda $R$, Ina $Y$, Suzuki $K$, Ohno T. Effects of benidipine and candesartan on kidney and vascular function in hypertensive dahl rats. Hypertens Res 2003; 26: 569-576.

Supplementary Information accompanies the paper on Hypertension Research website (http://www.nature.com/hr) 